\title{
Ejaculation in testicular cancer patients after post- chemotherapy retroperitoneal lymph node dissection
}

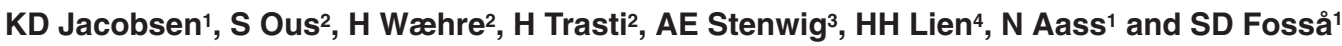 \\ Departments of ${ }^{1}$ Medical Oncology and Radiotherapy, ${ }^{2}$ Surgical Oncology, ${ }^{3}$ Pathology and ${ }^{4}$ Radiology, The Norwegian Radium Hospital, 0310 Oslo, Norway
}

Summary The purpose of this study was to evaluate fertility after different types of post-chemotherapy retroperitoneal lymph node dissection (RPLND). During 1980-1994, 192 patients with metastatic testicular cancer underwent post-chemotherapy RPLND with a gradual shift from modified bilateral template RPLND to nerve-sparing RPLND. Modified bilateral template RPLND was done in 92\% of the patients operated during 1980-1984 as compared to 16\% during 1989-1994. Pre- and post-treatment fertility was assessed by microscopic sperm analysis, determination of serum FSH and information on ejaculation and paternity. There was no significant difference of the survival rates between the three treatment periods. Antegrade ejaculation was preserved in $11 \%$ of the patients after modified bilateral template RPLND as compared to $89 \%$ after the nerve-sparing operation technique. The median ejaculatory volume decreased post-operatively, serum $\mathrm{FSH}$ increased and sperm density remained unchanged. Fifty-six patients attempted fatherhood after their treatment, and 27 fathered at least one child after an observation-time of 55 months, nine of them by assisted fertilization. Patients with initially advanced testicular cancer but limited residual retroperitoneal masses after induction chemotherapy can safely undergo limited post-chemotherapy RPLND as a part of multimodality treatment. After nerve-sparing RPLND antegrade ejaculation is preserved in $89 \%$ of the patients though the ejaculatory volume decreases after RPLND. Post-treatment fatherhood can be achieved in at least $50 \%$ of the patients attempting paternity.

Keywords: testicular cancer; nerve-sparing RPLND; fertility

During recent years increasing attention has been devoted to longterm toxicity and quality of life of patients treated for testicular cancer (Moynihan, 1987; Roth et al, 1988; Aass et al, 1990; Bissett et al, 1990), fertility being an important dimension of psychosocial well-being and post-treatment morbidity.

Spermatogenesis is often transiently inhibited by modern standard chemotherapy (Drasga et al, 1983; Berthelsen, 1984; Brenner et al, 1985; Lange et al, 1987) with gradual recovery 10-18 months after discontinuation of chemotherapy. After retroperitoneal lymph node dissection (RPLND), 'dry ejaculation' represents a major risk of infertility, especially if this operation has to be done in patients with extended retroperitoneal metastases (Hermanek and Sigel, 1982; Wood et al, 1982; Lange et al, 1984; Fosså et al, 1991). PostRPLND dry ejaculation is due to the resection of sympathetic nerves entering the superior hypogastric plexus (Jewett et al, 1988). After the original bilateral template RPLND almost all patients suffered from post-operative dry ejaculation, even if done as modified RPLND thus sparing the contralateral iliac region below the iliac bifurcation (Whitmore, 1982). Early in the 1980s unilateral template RPLND was developed (Fosså et al, 1984a; Pizzocaro et al, 1985; Weissbach et al, 1985). After this operation antegrade ejaculation remained in $80 \%$ of the patients. During the mid 1980s Donohue et al (1990) introduced nerve-sparing RPLND in the treatment of testicular cancer patients.

Unilateral and nerve-sparing RPLND techniques were primarily developed for patients with no or limited retroperitoneal tumour

\section{Received 28 May 1998}

Revised 24 October 1998

Accepted 13 October 1998

Correspondence to: SD Fosså manifestations and were mostly applied as diagnostic means in testicular cancer patients without preceding chemotherapy. During the 1980s it became evident that more limited techniques of RPLND, such as unilateral (Fosså et al, 1985a) or nerve-sparing (Jewett et al, 1988) RPLND, might be sufficient. Some institutions even used unilateral RPLND techniques as post-chemotherapy surgical procedures. Other institutions performed resection of residual macroscopic tumours rather than a formal RPLND (Hendry et al, 1993).

In the present paper we summarize the Norwegian Radium Hospital's (NRH) experience with post-chemotherapy RPLND from 1980 to 1994 . The aim was to investigate the impact of different types of RPLND on ejaculatory function and thus on fertility.

\section{MATERIALS AND METHODS}

\section{Patients}

From 1980 to 1994 about 400 patients with testicular cancer underwent RPLND at the NRH, Oslo. A total of 192 operations were performed after cisplatin-based induction chemotherapy in patients presenting with metastases. Survival data are given for these 192 patients. The analysis of ejaculatory function and fertility comprises 174 men, excluding 13 patients who had died at the time of investigation (March 1996) and for whom the medical records did not contain any information about post-RPLND ejaculation (Table 1). Also excluded were four patients with severe psychological disturbances from whom no valid information could be obtained as regards ejaculatory function and one patient who was lost to follow-up without any information on ejaculatory function and fertility in his medical record. The patients' initial staging 
Table 1 Demographics

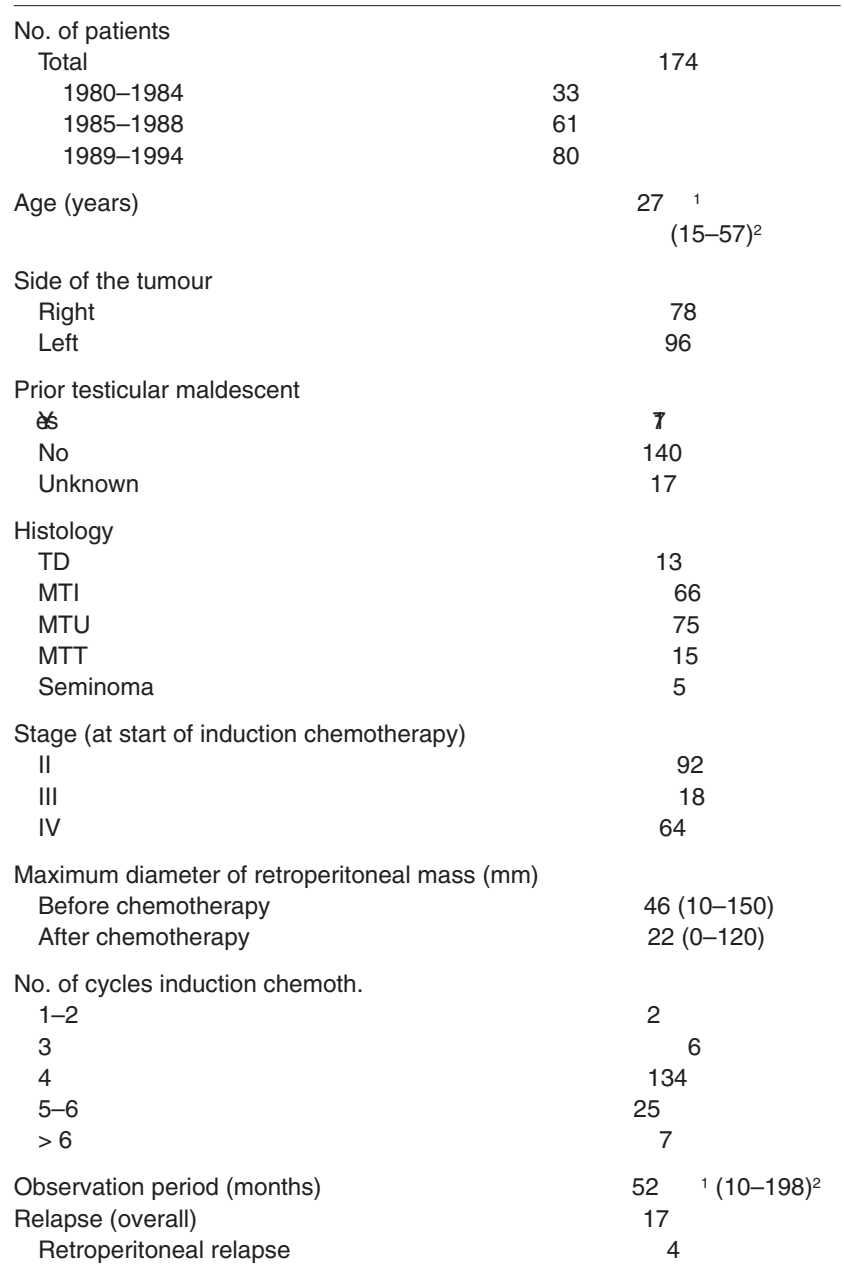

${ }^{1}$ Median; ${ }^{2}$ (range). TD = terattenendiated (teratoma); MTI = malignant teratoma intermediate (teratocarcinoma); MTU = malignant teratoma undiferentiated (embryonal carcinoma); MTT = malignant teratoma trophoblastic (choriocarcinoma).

was done according to the Royal Marsden classification system based on clinical examination, thoraco-abdominal computerized tomography (CT) and measurements of serum choriogonadotropin (HCG) and alpha fetoprotein (AFP).

\section{Treatment principles}

Cisplatin-based induction chemotherapy was given to all patients wit $\mathrm{h} \geq$ IIB non-seminomatous testicular cancer o $\geq$ IICrseminoma. Post-chemotherapy RPLND was to be done 4-10 weeks after induction chemotherapy in non-progressing patients who had initially presented with retroperitoneal tumour masses, even though these had completely regressed after cytostatic treatment as evidenced by abdominal CT (Fosså et al, 198 a). Entil about 1985 patients with advanced seminoma were included in postchemotherapy RPLND treatment, but this policy was gradually abandoned thereafter (Fosså et al, 1987, 1988).
Table 2 đar of retroperitoneal surgery and type of RPLND (A), stage (B) and size of retroperitoneal mass (C) (192 patients)

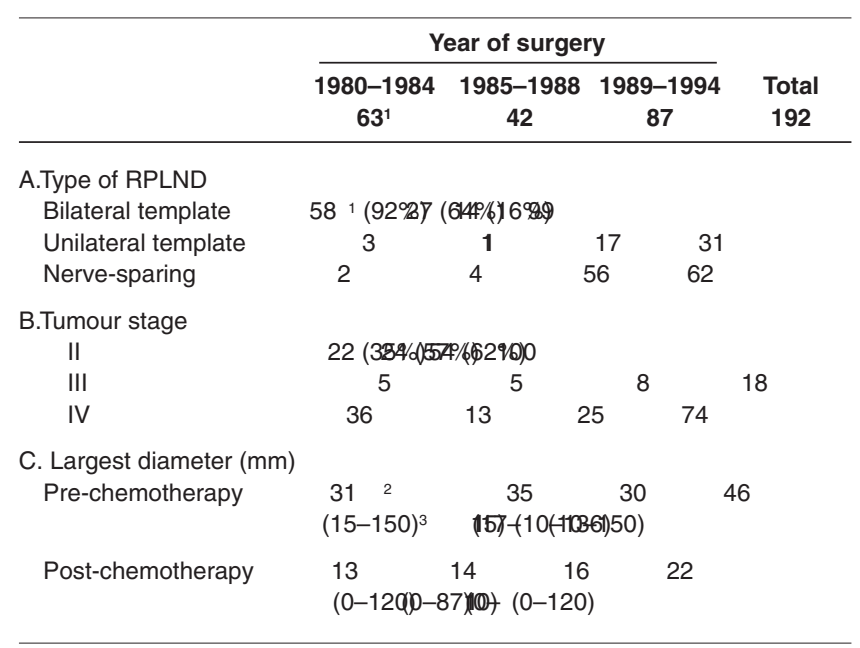

${ }^{1}$ Number of patients; ${ }^{2}$ median; ${ }^{3}$ (range).

\section{Chemotherapy}

From 1978 and the early 1980s, induction chemotherapy consisted of 4-6 cycles with the PVB regimen (cisplatin, vinblastine, bleomycin) (Narayan et al, 1982; Fosså et al, 1988; Aass et al, 1991). A few patients from 1980 to 1981 also received adriamycin as a component of consolidation treatment. From 1985, vinblastine was gradually substituted by VP-16 (bleomycin, etoposide, cisplatin [BEP]). From 1988, patients were included in international randomized trials evaluating the e f fect of carboplatin and ifosfamide in patients with germ cell tumours (Loehre r, 1990 Bajorin et al, 1993; Bosl and Motze r, 1997).

\section{Surgical procedures for RPLND}

Three types of RPLND were carried out during the 15-year period (Table 2).

\section{Modified bilateral template RPLND}

This operation was routine from 1980 to 1984 and followed the recommendations of Whitmore (1979): excluding the great vessels, all retroperitoneal soft tissue was excised as an anatomical template.

\section{Unilateral template RPLND}

From 1985 to 1988 , resection of retroperitoneal tissue was confined to the ipsilateral side of the aortic midline in patients with retroperitoneal tumour disease confined to one template.

During nerve-sparing RPLND the sympathetic nerve fibres were carefully dissected out, identified and preserved, at least on one side (Donohue et al, 1990). Unilateral template nerve-sparing RPLND (Figure 1) was introduced in 1992 by one of the authors (SO) for patients with tumours confined to the ipsilateral template.

Va a midline abdominal incision the peritoneum was incised from the foramen of Winslo w, distally around the caecum and proximall $y$, along the left side of the mesenteric root, to the superior mesenteric arter $y$. The right colon and the mesentery of the small intestine were sharply mobilized o ff the thin fascia covering the retroperitoneal compartment and placed on the patient 


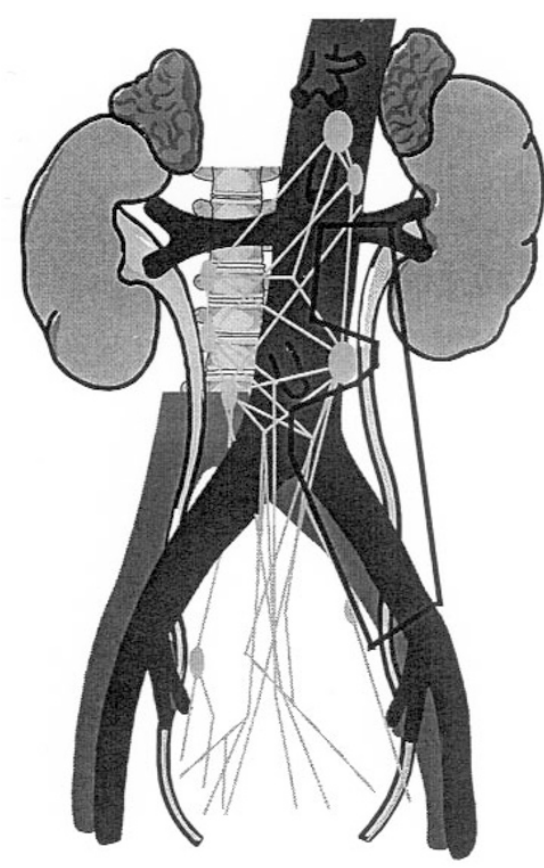

Figure 1 Schematic presentation of the unilateral nerve-sparing RPLND. The pre-aortic sympathetic ganglion close to the origin of the inferior mesenteric artery is attempted to be preserved together with the hypogastric nerve bundle

The pancreas and the third part of duodenum were mobilized. With the left-sided template, the inferior mesenteric artery was routinely divided near to the aorta and the mesentery of the sigmoid colon was mobilized. The inferior mesenteric vein was usually preserved.

The nerve-sparing dissection commenced with the visual identification of the hypogastric nerves on the anterior surface of the aortic bifurcation, beneath the thin subperitoneal fascia covering aorta and the retroperitoneal compartment. The nerve bundles were safeguarded with a rubberband and followed proximally, until they diverged to their sides of origin. The landmark was usually identifiable at a ganglion at the pre-aortic level just distal to the origin of the inferior mesenteric artery. This ganglion was preserved. The nerve trunks belonging to the template of the ipsilateral template were removed. No further attempt was performed to dissect tissues on the opposite side.

Starting at the common iliac artery the tissue covering the great vessels was ligated, divided, mobilized and rolled off the circumference of the vessels proceeding stepwise proximally along the anterior midline of the vessels. Lumbal vessels were visualized, secured and divided. Following complete mobilization of the great vessels from the posterior abdominal wall within the appropriate template, all soft tissue was removed from the retroperitoneal space and the anterior spinous ligament. The root of the mesentery was repositioned and the peritoneum covering the posterior abdominal wall repaired with interrupted sutures.

\section{Post-RPLND management}

No further treatment was given in patients with complete postchemotherapy resections if the histology revealed complete necrosis/fibrosis or mature teratoma. Patients with viable cancer tissue in the resection specimen received three cycles of postoperative adjuvant chemotherapy (Hollender et al, 1997).

\section{Follow-up}

All patients were seen at the NRH's outpatient department or at a local hospital with 2-6 months' intervals during the first 3-5 years, with no subsequent follow-up visits at the NRH. In all patients, a post-operative abdominal CT was performed about 2 months after RPLND. Routine follow-up investigations comprised clinical examination, measurements of serum HCG, AFP and regular chest X-rays. CT examinations were only performed when clinically indicated.

\section{Assessment of spermatogenesis and fertility}

Before 1985 only those patients were assessed for pre-treatment fertility who spontaneously expressed plans for post-treatment paternity and in whom pre-treatment semen cryopreservation was considered. From 1985, information of testicular maldescent, previous fatherhood and involuntary infertility was routinely obtained. Evaluation of fertility was routinely done in all patients at the time of the initial referral to the NRH (2-3 weeks after unilateral orchiectomy, which usually was performed at the patient's local hospital). The serum hormone levels of luteinizing hormone ( $\mathrm{LH})$, follicle-stimulating hormone (FSH) and testosterone were measured. Light microscopic sperm cell analysis in the post-masturbation seminal fluid obtained after 3 days' sexual abstinence was done assessing sperm cell density $\left(\times 10^{6} \mathrm{ml}^{-1}\right)$, mobility (grade 1-4) and motility (\% mobile sperm cells). For the sake of simplification only sperm cell density will be considered in the present study. Whenever possible, serum hormone analyses and sperm analysis were repeated 1 and 3 years after discontinuation of all treatment. In addition, at the follow-up visits patients were to be asked about their post-operative ejaculatory function, their partners' eventual pregnancies and about involuntary infertility. If indicated, couples with infertility problems were referred to the University Infertility Clinic of the National Hospital, Oslo.

For the purpose of the study, a questionnaire was mailed to 165 living patients in March 1996 asking them for more recent information about post-treatment fatherhood, infertility and ejaculatory function. The patients were asked to categorize their ejaculation as 'normal' (antegrade), 'varying' (sometimes antegrade, sometimes 'dry ejaculation'), or as 'dry ejaculation' (never seminal fluid per urethram). The completed form, which represented the principal source of this study's outcome parameters, was returned by 155 patients $(94 \%)$. For the ten patients who did not respond to the mailed questionnaire, and for nine patients who had died by the time of investigation, comparable information from the medical record was considered in the analysis.

\section{Statistical analysis}

The data were analysed by the PC-version 6.0 of the SPSS using standard statistics (median, mean, range, $\chi^{2}$ test, Student's $t$-test). Due to the small number of paired results from sperm counts we decided to analyse all available pre- and post-treatment samples as independent parameters. Cancer-specific survival was assessed by the Kaplan-Meier method, evaluating differences between curves by the log-rank test. A $P$-value $<0.05$ was regarded as statistically significant.

\section{RESULTS}

\section{Performance of RPLND}

During 1980-1984, 58 of 63 RPLNDs (92\%) were performed as bilateral template operations (Table 2). This percentage had 


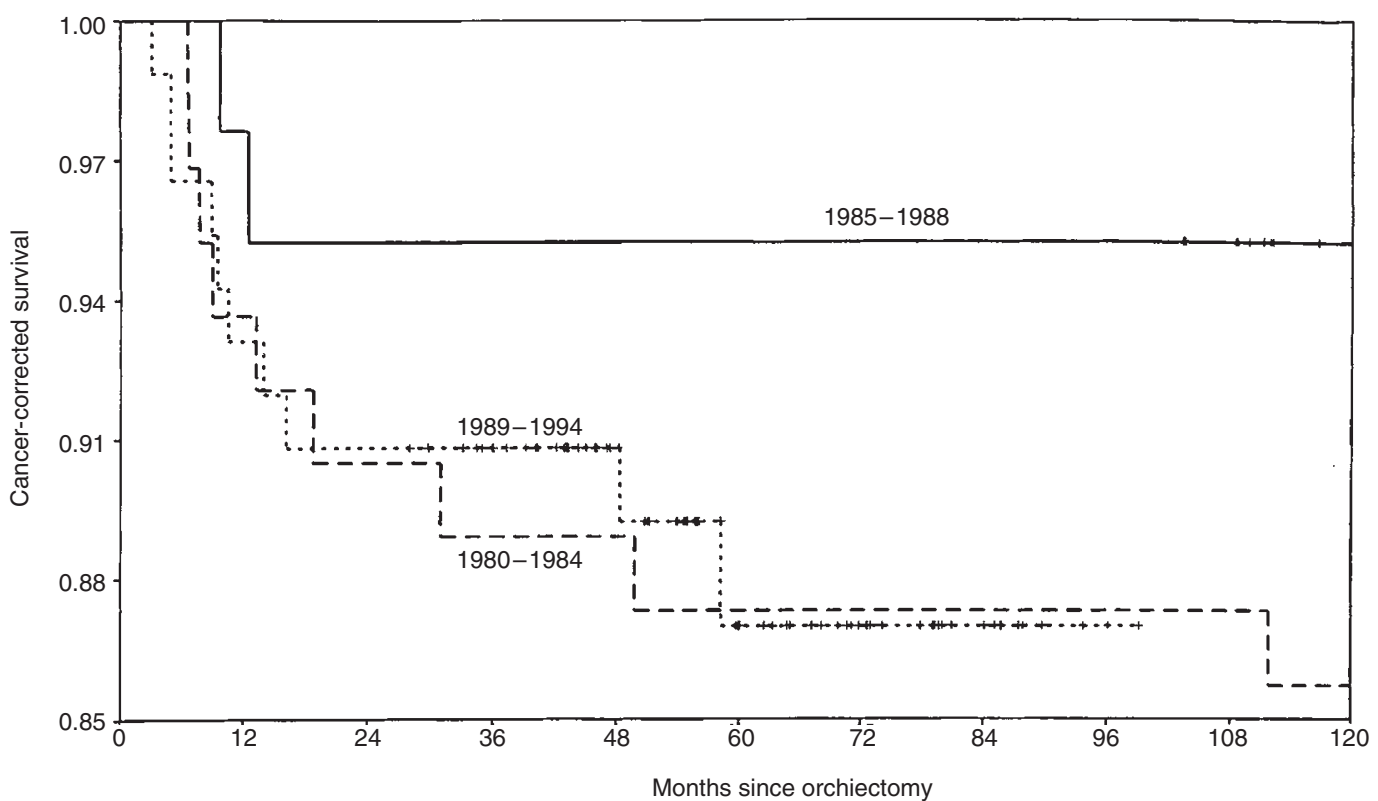

Figure 2 Cancer-specific survival in patients with metastatic testicular cancer undergoing post-chemotherapy RPLND at the NRH (- - - 1980-1984, (-) 1985-1988, (. . . .) 1989-1994

decreased to $16 \%$ (14 of 87 ) during the third period. This reduction was not related to less advanced retroperitoneal tumour masses in the third period, though the percentage of stage II patients had increased gradually (Table 2B and C).

\section{Relapse and survival}

In 30 of the 192 patients, the malignancy relapsed after RPLND. In 26 of these patients, residual viable cancer was found in the operation specimen; residual mature teratoma was demonstrated in four patients. In seven of these 26, recurrent retroperitoneal tumour masses were found at the time of relapse. Three of the seven patients had undergone modified bilateral template RPLND, one had had unilateral template RPLND and three patients were operated on by a nerve-sparing technique. The recurrent retroperitoneal tumours were resected in four patients, the histology showing viable malignant tumour tissue in one of them. In the three remaining patients with retroperitoneal relapse, all of them after nerve-sparing RPLND, the histology of the recurrent tumour was 'mature teratoma'. The former patient subsequently died of the germ cell malignancy, whereas the three other patients remain tumour-free. With a median observation time of 96 months (range 27-205) for surviving patients the cancer-specific survival was not significantly correlated with the period of RPLND (Figure 2).

Table 3 Ejaculation and years for RPLND

\begin{tabular}{lcccc}
\hline & \multicolumn{3}{c}{ Year of surgery } & \\
\cline { 2 - 4 } Ejaculation & $\mathbf{1 9 8 0 - 1 9 8 4}$ & $\mathbf{1 9 8 5 - 1 9 8 8}$ & $\mathbf{1 9 8 9 - 1 9 9 4}$ & Total \\
\hline Antegrade & $17(\mathbf{2 8 \%})$ & $2(6 \%)$ & $63(79 \%)$ & 82 \\
Variable & $5(8 \%)$ & $5(15 \%)$ & $6(7 \%)$ & 16 \\
'Dry' & $39(64 \%)$ & $26(79 \%)$ & $11(14 \%)$ & 76 \\
Total & 61 & 33 & 80 & 174 \\
\hline
\end{tabular}

Antegrade ejaculation was at least partially preserved in 17 of the $61(28 \%)$ patients operated on during the first 5 years as compared to two of 33 patients $(8 \%)$ and 63 of 80 patients $(79 \%)$ in the second and third period respectively (Table 3 ).

Sixty-seven of 89 patients (75\%) complained of dry ejaculation after bilateral modified template RPLND (Table 4). The comparable figures after nerve-sparing operations were three of $56(5 \%)$. Dry ejaculation occurred in six of 29 patients $(21 \%)$ operated on by unilateral template RPLND, three of them operated due to right-sided and three operated for left-sided tumours.

The median ejaculatory volume decreased from $4.4 \mathrm{ml}$ before RPLND (64 patients) to $2.5 \mathrm{ml}$ after the operation (26 patients) $(P<0.001$, Table 5$)$. With the reservation of small numbers, the reduction of the ejaculatory volume was least after nerve-sparing RPLND as compared to the template RPLND techniques without nerve-sparing. The sperm cell density remained unchanged.

Fifty-six of the 157 evaluable patients (36\%) attempted to achieve fatherhood after their treatment (Table 6). Of the 35 patients with antegrade/variable ejaculation 18 were successful, two of them by fertilization using the patient's post-treatment sperm cells. Only nine of the 21 patients with dry ejaculation who planned paternity achieved fatherhood after their treatment, seven patients by assisted fertilization using the pre-treatment sperm cells from the sperm bank, and two patients after treatment with sympathomimetic agents.

Table 4 Ejaculation and type of RPLND

\begin{tabular}{lcccc}
\hline & \multicolumn{3}{c}{ Type of surgery } & \\
\cline { 2 - 4 } Ejaculation & $\begin{array}{c}\text { Modified bilateral } \\
\text { template }\end{array}$ & $\begin{array}{c}\text { Unilateral } \\
\text { template }\end{array}$ & Nerve-sparing & Total \\
\hline Antegrade & $10(11 \%)$ & $22(76 \%)$ & $50(89 \%)$ & 82 \\
Variable & $12(13 \%)$ & $1(3 \%)$ & $3(5 \%)$ & 16 \\
'Dry' & $67(75 \%)$ & $6(21 \%)$ & $3(5 \%)$ & 76 \\
Total & 89 & 29 & 56 & 174 \\
\hline
\end{tabular}


The policy of performing nerve-sparing post-chemotherapy RPLND, whenever possible, has been followed by antegrade or variable ejaculation in $86 \%$ of the patients operated from 1989 to 1994 , and antegrade ejaculation is preserved in $95 \%$ of the patients by nerve-sparing technique. However, the seminal fluid volume decreases as compared to the pre-treatment situation. The fact that with a median observation time of 55 months about one-third of the patients have attempted fatherhood underlines the clinical importance of preservation of antegrade ejaculation. An additional advantage of preserved antegrade ejaculation is that the patients' post-treatment semen may be used for assisted fertilization if fatherhood can not be achieved by 'natural means'.

Hendry et al (1992) had similar favourable results as concerns preservation of antegrade ejaculation when removing a residual mass rather than performing a formal RPLND. No data are so far available which would indicate the superiority of either treatment. At present we recommend institutions to choose the type of operation they feel most confident with.

After post-chemotherapy nerve-sparing RPLND there might be slightly increased risk of a retroperitoneal recurrence. Safe follow-up of these patients is essential and requires the close cooperation between clinicians and radiologists. The interpretation of post-RPLND abdominal CTs requires thorough knowledge of the typical visualization of the post-operative anatomy. The performance of unilateral template RPLND, but in particular of nervesparing RPLND, requires experience in the technical aspects of the operation, understanding of the tumour biology of advanced testicular cancer after induction chemotherapy and a close cooperation with the responsible oncologist and pathologist. Only major cancer centres will probably gain sufficient experience which lead to optimal results.

The present report leads to the following conclusions:

1. Modern treatment of chemotherapy of testicular cancer has to take into account that at least one-third of the patients want to father a child after their treatment.

2. Post-chemotherapy RPLND can safely be done by nervesparing techniques in most patients with limited residual retroperitoneal tumour masses.

3. Nerve-sparing post-chemotherapy RPLND preserves antegrade ejaculation in about $90 \%$ of the patients and enables post-treatment paternity in at least $50 \%$ of those who attempt fatherhood after their treatment.

4. The close cooperation between oncologists, surgeons, pathologists and radiologists is a condition sine qua non in the fertility-preserving approach nerve-sparing RPLND.

\section{ACKNOWLEDGEMENTS}

The authors want to thank John P Donohue, MD, PhD for introducing the nerve-sparing operation technique for the surgical staff. The Norwegian Cancer Society is acknowledged for financing the project.

\section{REFERENCES}

Aass N and Fosså SD (1988) Paternity in young patients with testicular cancer expectations and experience. Prog Clin Biol Res 269: 481-491

Aass N, Fosså SD, Aas M and Lindegaard MW (1990) Renal function related to different treatment modalities for malignant germ cell tumours. Br J Cancer 62: $842-846$
Aass N, Fosså SD, Theodorsen L and Norman N (1991) Prediction of long-term gonadal toxicity after standard treatment for testicular cancer. Eur J Cancer 27: 1087-1091

Bajorin DF, Sarosdy MF, Pfister DG, Mazumdar M, Motzer RJ, Scher HI, Geller NL, Fair WR, Herr H, Sogani P, Sheinfeld J, Russo P, Vlamis V, Carey R, Vogelzang NJ, Crawford ED and Bosl GJ (1993) Randomized trial of etoposide and cisplatin versus etoposide and carboplatin in patients with good-risk germ cell tumors: a multiinstitutional study. J Clin Oncol 11: 598-606

Berthelsen JG (1984) Sperm counts and serum follicle-stimulating hormone levels before and after radiotherapy and chemotherapy in men with testicular germ cell cancer. Fertil Steril 41: 281-286

Bissett D, Kunkeler L, Zwaneburg L, Paul J, Gray C, Swan IRC, Kerr DJ and Kaye SB (1990) Long-term sequelae of treatment for testicular germ cell tumours. $\mathrm{Br}$ J Cancer 62: 655-659

Bosl GJ and Motzer RJ (1997) Testicular germ-cell cancer. Review Art. N Engl J Med 337: 242-253

Brenner J, Vugrin D and Whitmore WF Jr (1985) Effect of treatment on fertility and sexual function in males with metastatic nonseminomatous germ cell tumors of testis. Am J Clin Oncol 8: 178-182

Donohue JP, Foster RS, Rowland RG, Bihrle R, Jones R and Geier G (1990) Nervesparing retroperitoneal lymphadenectomy with preservation of ejaculation. J Urol 144: 287-292

Drasga RE, Einhorn LH, Williams SD, Patel DN and Stevens EE (1983) Fertility after chemotherapy for testicular cancer. J Clin Oncol 1: 179-183

Fosså SD, Klepp O, Ous S, Lien HH, Stenwig AE, Åbyholm T and Kaalhus O (1984a) Unilateral retroperitoneal lymph node dissection in patients with nonseminomatous testicular tumor in clinical stage. I. Eur Urol 10: 17-23

Fosså SD, Klepp O, Ous S, Lien HH, Stenwig JT, Abeler V, Eliassen G and Høst H (1984b) Multi-modality treatment in males with advanced malignant germ cell tumours. II. Experience with surgery and radiotherapy following cisplatinumbased chemotherapy. Scand J Urol Nephrol 18: 21-26

Fosså SD, Ous S, Åbyholm T and Loeb M (1985a) Post-treatment fertility in patients with testicular cancer. I. Influence of retroperitoneal lymph node dissection on ejaculatory potency. Br J Urol 57: 204-209

Fosså SD, Ous S, Åbyholm T, Norman N and Leob M (1985b) Post-treatment fertility in patients with testicular cancer. II. Influence of cisplatin-based combination chemotherapy and of retroperitoneal surgery on hormone and sperm cell production. Br J Urol 57: 210-214

Fosså SD, Borge L, Aass N, Johannessen NB, Stenwig AE and Kaalhus O (1987) The treatment of advanced metastatic seminoma: Experience in 55 cases. J Clin Oncol 5: 1071-1077

Fosså SD, Aass N and Kaalhus O (1988) Testicular cancer in young Norwegians. Surg Oncol 39: 43-63

Fosså SD, Aass N, Ous S and Waehre H (1991) Long-term morbidity and quality of life in testicular cancer patients. Scand J Urol Nephrol Suppl 138: 241-246

Fosså SD, Qvist H, Stenwig AE, Lien HH, Ous S, Giercksky KE (1992) Is postchemotherapy retroperitoneal surgery necessary in patients with nonseminomatous testicular cancer and minimal residual tumor masses? J Clin Oncol 10: 569-573

Hendry WF, A'Hern RP, Hetherington JW, Peckham MJ, Dearnaley DP and Horwich A (1993) Para-aortic lymphadenectomy after chemotherapy for metastatic nonseminomatous germ cell tumours: Prognostic value and therapeutic benefit. Br J Urol 71: 208-213

Hermanek P and Sigel A (1982) Necessary extent of lymph node dissection in testicular tumours. A histopathological investigation. Eur Urol 8: 135-144

Hollender Å, Stenwig EA, Ous S and Fosså SD (1997) Survival of patients with viable malignant non-seminomatous germ cell tumour persistent after cisplatinbased induction chemotherapy. Eur Urol 31: 141-147

Jewett MAS, Kong YSP, Goldberg SD, Sturgeon JFC, Thomas GM, Alison RE and Gospodarowicz MK (1988) Retroperitoneal lymphadenectomy for testis tumor with nerve sparing for ejaculation. J Urol 139: 1220-1224

Lampe H, Horwich A, Norman A, Nicholls J and Dearnaley DP (1997) Fertility after chemotherapy for testicular germ cell cancers. J Clin Oncol 15: 239-245

Lange PH, Narayan P and Fraley EE (1984) Fertility issues following therapy for testicular cancer. Sem in Urol 2: 264-274

Lange PH, Chang WY and Fraley EE (1987) Fertility issues in the therapy of nonseminomatous testicular tumors. Urol Clin North Am 14: 731-747

Loehrer PJ Sr (1990) Ifosfamide in testicular cancer. Semin Oncol 17: 2-5

Moynihan C (1987) Testicular cancer: the psychosocial problems of patients and their relatives. Cancer Surveys 6: 477-510

Narayan P, Lange PH and Fraley EE (1982) Ejaculation and fertility after extended retroperitoneal lymph node dissection for testicular cancer. J Urol 127: $685-688$ 
Pizzocaro G, Salvioni R and Zanoni F (1985) Unilateral lymphadenectomy in intraoperative stage I non-seminomatous germinal testis cancer. J Urol 134: 485-489

Roth BJ, Greist A, Kubilis PS, Williams SD and Einhorn LH (1988) Cisplatin-based combination chemotherapy for disseminated germ cell tumors: long-term follow-up. J Clin Oncol 6: 1239-1247

Weissbach L, Boedefeld EA and Oberdörster W (1985) Modified RLND as a means to preserve ejaculation. In: Testicular Cancer, Khoury S, Kuss R, Murphy GP, et al (eds), pp. 323-334. Alan R. Liss: New York
Whitmore WF Jr (1979) Surgical treatment of adult germinal testis tumors. Semin Oncol 6: 55-68

Whitmore WF Jr (1982) Surgical treatment of clinical stage I nonseminomatous germ cell tumors of the testis. Cancer Treat Rep 66: 5-10

Wood DP, Herr HW, Heller G, Vlamis V, Sogani PC, Motzer RJ, Fair WR and Bosl GJ (1982) Distribution of retroperitoneal metastases after chemotherapy in patients with non-seminomatous germ cell tumors. J Urol 148: 1812-1816 